\title{
Medicine as Message: Caregiving, Illness Deception, and the Cultural Evolution of Harmful Treatments
}

\author{
Mícheál de Barra, Daniel Cownden $\mathcal{G}$ Fredrik Jansson
}

Mícheál de Barra (Corresponding author):

mdebarra@gmail.com

Instituite of Applied Health Sciences, University of Aberdeen

Aberdeen AB25 2ZD, UK

Daniel Cownden:

dcownden@gmail.com

Institute of Infection, Immunity and Inflammation, University of Glasgow

Glasgow G12 8TA, UK

Fredrik Jansson:

fredrik.jansson@su.se

Centre for the Study of Cultural Evolution, Stockholm University

SE-106 91 Stockholm, Sweden

Division of Applied Mathematics, Mälardalen University

SE-721 23 Västerås, Sweden

Mícheál de Barra funded by the Prof Roy Weir Career Development. Fredrik Jansson was funded by the Fellowship and Knut and Alice Wallenberg Foundation (grant number 2015.0005). These funders had no influence on the study design; in the collection, analysis and interpretation of data; in the writing of the report; or in the decision to submit the article for publication.

Word count: 7,927 


\section{Medicine as Message: Caregiving, Illness \\ Deception, and the Cultural Evolution of Harmful Treatments}

\section{Abstract}

Why did harmful medical treatments like bloodletting persist for centuries? We propose the following solution to this important cultural evolution question: Illness or injury often leaves people incapacitated for long periods. Caregiving - the provision of food, shelter etc. - is an important medical practice that enables ill or injured people and their dependents to survive these periods. However, caregiving is vulnerable to exploitation via illness deception whereby people feign illness in order to gain access to care. Harmful treatments helped to solve the problem of illness deception because only individuals who stand to gain substantially from care will accept the treatment. To investigate the plausibility of this theory we develop a mathematical model using evolutionary game theory and invasion analysis. We show that decreasing the benefit of caregiving via aversive and unpleasant medical treatments can counter-intuitively increase the range of conditions where caregiving is evolutionarily viable. Thus poisoning, cannibalism, emetics, bloodletting and other such treatments may be solutions to the problem of allocation of resources to people with unclear need.

Keywords: cultural evolution; medical anthropology; sick role; iatrogenic disease; evolutionary medicine; cooperation. 


\section{Introduction}

The idea that medicine should be unpleasant and aversive is well rooted in the English language. To take ones' medicine is synonymous with enduring a deserved painful or unpleasant experience. Cheats who are themselves cheated get a taste of their own medicine. This reputation is well earned: historical medical treatments were often repugnant, dangerous, taboo breaking or painful. Widespread procedures included ingestion of repugnant substances like animal wastes, bird nests and human flesh; ingestion of poisons, emetics, diuretics; surgical procedures like bloodletting, cupping (removal of blood by means of a vacuum), the reopening of partially healed wounds; and forced feeding or food/water restrictions (Chagnon, 2013; Edgerton, 1992; Sugg, 2008; Wootton, 2006). Treatments like blood-letting have been documented in 60 cultural/ethnic groups and existed in all continents bar Antarctica (Miton \& Mercier, 2015). Within the history of medicine, the idea that a substantial proportion of pre-20th century western medicines were ineffective or harmful is uncontroversial (Hardy, 2006; Wootton, 2006).

Given the paucity of effective treatments and abundance of unpleasant or harmful treatments, the long-term popularity of medicine is surprising since all else being equal, cultural innovations that result in greater efficiency, wealth or health tend to spread more successfully than innovations that make life poorer or more difficult (Boyd, Richerson, \& Henrich, 2013). It is also puzzling that these medical treatments should be so unpleasant. Patients who opted for a warm bath over bloodletting sacrificed no therapeutic value. Doctors who prescribed foot massages over emetics would have had happier and perhaps healthier patients. But rather than evolving towards gentle, pleasant, or comforting treatments, the medicine that persisted was invasive, macabre and painful - often theatrically so.

One medical intervention, however, is ancient, common, and beneficial to the patient: simple caregiving. Caregiving involves keeping patients dry, warm and clean, and providing food and water when necessary. Caregiving may also entail releasing people from or taking over their 
ordinary duties and obligations. This may include providing for dependents. Cross-cultural research indicates that this kind of care is both essential and widespread. Indeed, some anthropologists have argued that the human way of life is premised on the availability of caregiving (Kaplan, Hill, Lancaster, \& Hurtado, 2000; Sugiyama \& Chacon, 2000). In smallscale societies, people are often incapacitated by illness or injury and spend protracted periods of time unable to provide for themselves (Hill \& Hurtado, 2009). For example, Sugiyama (2004) found that $90 \%$ of Shiwiar - forager-horticulturists in the Ecuadorian Amazon - had spent a fortnight or longer incapacitated. Sixty percent of people fared even worse, spending a month or more unable to forage for themselves or their dependants. Without caregiving from friends and family, an illness or injury of this duration is fatal. When people are disabled by disease, relatives provide care and take over gardening tasks, sometimes for long periods. In the Shiwiar, as in other groups, people can survive these long periods of illness because friends and family transfer time, energy and food resources to the sick. Ancient remains also indicate that people often survived injury and illness; caregiving has long been available to those in need (Hublin, 2009). There is also some suggestion of caregiving across species (Byrne \& Stokes, 2003; Fabrega, 2011). Today about one in seven Americans provide care to a friend or family member who is ill or disabled in a given year (Marks, 1996).

This caregiving is not cost free. Sugiyama and Chacon describe how food provisioning to injured parties often lasts for weeks (2000), a substantial transfer in environments where food is not abundant. In the Tsimane, forager-horticulturists supply valued food and medicine as well as childcare to relatives who appear incapacitated (Gurven, Stieglitz, Hooper, Gomes, \& Kaplan, 2012). Sugiyama (2004) tells how "two informants reported that they jointly maintained [a sick woman's] gardens for three months, but stopped when they could no longer sustain the work necessary to do so and adequately maintain their own gardens as well", illustrating how people are willing to take on substantial caregiving costs. In contemporary Western societies, people involved in long-term caregiving experience poorer health (Vitaliano, Zhang, \& Scanlan, 2003) and increased mortality risk (Perkins et al., 2012; Schulz \& Beach, 
1999), suggesting that caregiving costs remain important even when health insurance and/or public health care provision exist. Collectively, these studies demonstrate that caregiving had - and continues to have - important costs.

From an evolutionary perspective, these costs often constitute a wise investment; helping a sick relative through a period of incapacity can have a substantial effect on their and their offspring's mortality. Hamilton's criterion for the evolution of care is frequently met $(c<r b$, where $r$ is relatedness, $b$ is benefit to the sick, and $c$ is cost to carer). This accords with the cross-cultural and historical research discussed above - caregiving between relatives is common and important.

\section{Illness deception}

Caregiving, however, is open to exploitation via illness deception. From an evolutionary perspective, the problem is simple: the range of conditions where recipients should request care $(r c<b)$ is much broader than the range of conditions where donors should be willing to grant care $(c<r b)$. If illness were transparent - that is, donors could accurately estimate how much the recipient would benefit - then this would be of little consequence. Care could be granted only when it benefited inclusive fitness. However, health status is usually opaque. Many debilitating illnesses leave little visible sign upon the body, for example, back pain, hernia, kidney stones, gallstones, diabetes, Lyme disease, and brucellosis. Moreover, many people with visible aberrations (scarring, rashes, disfigurement) are not in any need of care. Even among people with true illnesses, it is difficult to estimate how much they will benefit from a given transfer of resources. There is good evidence that people exploit this ambiguity in order to access caregiving which the donor would not be willing to offer had they complete information about the recipient's disease state.

Hysteria, malingering, factitious disorder and somatization disorder are terms used to describe a cluster of related phenomena whereby people assume an ill social state without having 
underlying pathology. Malingering is usually used to described people who consciously fake the disease in order to escape some onerous obligation like fighting in a war. Hysteria, later termed conversion disorder, describes situations where the "deception" is not under conscious control; the individuals honestly believe themselves to be ill. Factitious disorder, like malingering, involves deliberate deception, but unlike malingering, the deception is not intended to release one from some specific unwanted duty. Rather, the person intends to gain access to the general benefits of the sick role. In reality, clear distinctions between these conditions are difficult to make. People differ in the degree to which they seek release from a specific duty versus the general emotional and practical benefits of caregiving, and in the degree to which the the deception is consciously planned and executed versus subconsciously motivated. Here we refer to any attempt to feign or exaggerate illness which may result in access to caregiving as illness deception, irrespective of whether the behaviour is unconsciously or consciously motivated, and irrespective of whether the scale of the deception is severe or more trivial.

Illness deception is common. In one survey of clinical neuropsychologists, $30 \%$ of personal injury cases and $33 \%$ of disability and worker's compensation cases were judged to "probably" involve malingering or symptom exaggeration. Illness deception was identified when, e.g., impairment was inconsistent with condition or there were discrepancies among records, self-report and observed behaviour (Mittenberg, Patton, Canyock, \& Condit, 2002). In the economic literature, meta-analyses have demonstrated a positive relationship between compensation benefits and both the number and duration of claims (Loeser, Henderlite, \& Conrad, 1995). Moreover, fluctuations in illness compensation claims appear unrelated to disease prevalence (Gun, 1990; Halligan, Bass, \& Oakley, 2003; Nicholson \& Martelli, 2007) and the introduction of compensation processes is associated with increasing pain reports and reduced treatment effectiveness (Rohling, Binder, \& Langhinrichsen-Rohling, 1995). Moreover, studies have demonstrated that actors can fool health professionals reasonably easily (Norman, Tugwell, \& Feightner, 1982); given that many diseases have inconsistent and 
non-observable symptoms this is unsurprising. Illness deception has also been documented in the historical record (see, e.g., Withey, 2013). As several authors argue (Finlay \& Syal, 2014; Steinkopf, 2015, 2016; Tiokhin, 2016), the fitness benefits associated with care may have acted as a selection pressure on symptom presentation and such a selection pressure may not always result in honest displays. Consistent with this there are speculative reports of illness deception in non-human animals.

\section{Aversive medicine maintains honesty}

Caregiving is an ancient and beneficial medical practice that enhances the inclusive fitness of both donor and recipient, but it is vulnerable to exploitation via illness deception. We show that decreasing the benefit of caregiving via aversive and unpleasant medical treatments can increase the range of conditions where caregiving is evolutionarily viable. This counter-intuitive result can be roughly understood as follows: in many situations where illness deception might undermine the viability of caregiving, a slight decrease in the benefit of the treatment can shift conditions so that illness deception is no longer viable, allowing caregiving to flourish. These added costs to receiving treatment keep communication honest by allowing caregivers to avoid the problem of distinguishing the ill from the illness deceivers, and by allowing those with hard-to-detect or easily-fabricated-yet-serious illnesses to credibly request care.

This result is possible because truly sick people have much more to gain from caregiving than someone engaged in illness deception. For someone with a significant illness, caregiving can prevent death. For someone with a minor illness or no disease, caregiving provides a lesser

benefit, for example, release from duty or additional food. From an evolutionary perspective, if the aversive treatment (e.g. bloodletting or emetics) is of the appropriate cost, then people will never engage in illness deception. In his discussion of symptoms-as-signals, Tiokhin (2016) independently arrives at a similar suggestion.

To better understand how aversive treatments operate to enable efficient caregiving, we 
develop a mathematical model. Models help to direct our attention to key assumptions, as well as suggest predictions that might be tested in future. Moreover, ineffective costly medicine is not a phenomenon with a single explanation. A formal model can help us to understand the specific circumstances where it does (and does not) apply.

\section{Model}

We will formulate an evolutionary model where, in short, individuals reproduce asexually, can be healthy or sick, and where they meet other individuals in random interactions. In these interactions, people have a strategy of whether to ask for help, at a cost to the helper (that causes reduction in fecundity) and a benefit (increasing fecundity) to the recipient, if provided, and whether to provide help when asked. Interactions are assorted, such that relatives meet at a certain frequency.

We use evolutionary game theory with fundamental ideas from invasion analysis (Maynard Smith \& Price, 1973) to explore the interaction of illness deception, harmful medicine and caregiving. Specifically, we are interested in the conditions under which providing help is a stable strategy and those where it is not. Our main question is whether the range of conditions where helping is evolutionarily viable can be increased through the introduction of aversive medicine. We will first specify the evolutionary model in further detail, then spell out the simplifying assumptions needed for our analytical strategy, and finally derive conditions for helping and asking strategies to be maintained in the population.

\section{Specification and Assumptions}

More specifically, an individual encounters the opportunity to make fraudulent requests for care (ask for help when sick) with frequency $f_{\mathrm{s}}$, and the opportunity to make honest requests for care (ask for help when healthy) with frequency $f_{\mathrm{h}}$. We assume that these 
frequencies are set at the population level, that is, they are the same for all individuals. Since every opportunity for an individual to request care when ill is paired with an opportunity for another individual to provide that help (conditional on the request being made ${ }^{1}$ ), an individual encounters the potential opportunities to provide help with the same frequencies: $f_{\mathrm{s}}$ to a sick individual and $f_{\mathrm{h}}$ to a healthy individual. When asked for help, an individual does not know whether the requester is sick or healthy.

Providing care entails a cost $c$. Receiving help gives a benefit $b_{\mathrm{s}}$ if the recipient is sick, and $b_{\mathrm{h}}$ if she is healthy. We assume throughout that the benefit of care when sick is greater than when healthy, $b_{\mathrm{s}}>b_{\mathrm{h}}$.

Finally, we assume that there is an assortative mechanism that produces a degree of relatedness $r$ between interacting individuals. Relatedness is here defined as the probability that an allele sampled from the actor will be identical by descent to an allele sampled from the recipient, and hence they will employ the same strategy. We will return to this assumption below.

To derive conditions for helping to be maintained in this model, the general idea is to consider the situations where there is a resident strategy at dynamical equilibrium and evaluate the initial growth rate of a mutant strategy in such an environment, the invasion fitness (see e.g. Brännström, Johansson, \& Festenberg, 2013). The success of the mutant strategy is then inferred by the growth rate when rare. As is common in invasion analysis, we incorporate the simplifying assumptions that the strategies interact within an infinite population, that reproduction is asexual, and that interaction occurs between pairs of strategies. Although these behaviours are cultural traits, our model focuses on the genetic fitness of people who engage in these behaviours. Later, we discuss how this genetic fitness might translate into cultural success.

Returning to the degree of relatedness $r$, suppose that there is a behaviourally relevant

\footnotetext{
${ }^{1}$ Note that these are ${ }^{*}$ opportunities* for people to make fraudulent or honest requests for care. Whether an individual will actually make or recieve a fraudulent or honest request depends on the strategy of the requester.
} 
allele that causes reduction in personal fecundity $c$ (for cost) while at the same time causing the fecundity of some other individuals to be increased by $b$ (for benefit). Hamilton (1963) showed that in the case of discrete, non-overlapping generations, this allele for a helping behaviour can spread provided that there is some assortative mechanism whereby individuals are more likely to interact with relatives. Specifically, helping behaviour will be favoured by natural selection precisely when $r b>c$, where $r$ is the relatedness between the actor and the recipient. While this seems relatively straightforward, it should be noted that many of the plausible assortative mechanisms that might cause interactants to be related, for example spatial structure coupled with limited offspring dispersal, can also serve to localise competition so that the benefits of cooperation are squandered in subsequent competition between relatives (West, Pen, \& Griffin, 2002). Here we ignore these issues and simply assume that an unspecified assortative mechanism produces a degree of relatedness $r$, and that the costs and benefits that we will consider are all in terms of a simple fecundity benefit within the context of discrete non-overlapping generations. This is a simplification, but since our aim is to illustrate the evolutionary potential of aversive medicine rather than to derive exact conditions for values of parameters (that lack empirical data), simplicity and clarity are more important.

To be clear, relatedness $r$ is thus an input parameter to the model, and is the same throughout the population (as in the signalling model by Maynard Smith, 1991). In a scenario with several strategies in the population, this could potentially have a large impact on the dynamics, if we can expect that the success of different strategies will influence $r$. In our analysis, however, we compare the fitness of residents with the same strategy only to mutants with another strategy, similar to the approach taken by Taylor and Frank (1996), where $r$ remains the same for a rare mutant (see also Gardner \& West, 2006, on the relative merits of approaches with closed models where $r$ is determined by demographic assumptions versus open models where it is allowed to vary independently). In fact, as will be obvious in the invasion analysis, $r$ is only relevant in the fitness equation for the mutant, so in our analysis, $r$ can be interpreted 
as the frequency with which mutants interact with individuals identical by descent, and $1-r$ as the frequency with which they interact with the rest of the population.

As mentioned earlier, while the person requesting care 'knows' which game they are playing, that is, whether they are healthy or sick, the person receiving the request does not (health status is opaque). The possible strategies in this game are thus composed of three components: 1) whether or not to request care when ill, 2) whether or not to request care when healthy and 3) whether or not to provide care when it is requested. This means that there are eight $\left(2^{3}\right)$ possible strategies, allowing for all possible combinations of the component parts of the strategies. However, three of these strategies weakly dominate the rest and so we limit our analysis to these three. The three dominant strategies are Deceptive Nonhelper, which will request care both when ill and healthy and does not provide care when asked, Honest Helper, which requests care only when truly ill and provides care when asked, and Deceptive Helper, which requests care both when ill and healthy and provides care when asked. Since there is only one non-helping strategy, and no honest non-helpers, we will henceforth refer to Deceptive Nonhelper simply as Nonhelper. (Weak domination means that any strategy outside of the set of Nonhelper, Honest Helper and Deceptive Helper, can only ever do as well as, but never better, than one of these dominating strategies, regardless of the population profile.)

The general equation for any of these strategies is 
Fitness increment $=$ Expected benefit of care when sick

- Expected cost of caring for sick

+ Expected benefit of care when healthy

- Expected cost of caring for healthy

$=f_{\mathrm{s}} b_{\mathrm{s}} \cdot P($ Receive care when sick $)$

$-f_{\mathrm{s}} c \cdot P($ Provide care for sick $)$

$+f_{\mathrm{h}} b_{\mathrm{h}} \cdot P($ Ask for and receive care when healthy $)$

$-f_{\mathrm{h}} c \cdot P($ Provide care for healthy)

In each of these expressions fitness is computed as the expected benefit or cost in the following four situations: asking for help as a sick person (which happens with frequency $f_{\mathrm{s}} P$ (Receive care) since all strategies ask when sick); being asked for help by a sick person (with frequency $f_{\mathrm{s}}$ if the strategy provides care); having the opportunity to ask for help as a healthy person (with frequency $f_{\mathrm{h}} P$ (Receive care) if the strategy asks for help when healthy); and potentially being asked for help by a healthy person (with frequency $f_{\mathrm{h}}$ if the strategy provides care and the recipient asks for it when healthy). We examine the fitness expressions for each strategy in turn.

We let $P_{\mathrm{N}}, P_{\mathrm{H}}$ and $P_{\mathrm{D}}$ denote the proportions in the population of Nonhelper, Honest Helper and Deceptive Helper strategies, respectively, and let $W_{\mathrm{N}}, W_{\mathrm{H}}$ and $W_{\mathrm{D}}$ denote their respective fitness. Then we can compute the fitness benefit for Nonhelpers as

$$
\begin{aligned}
\Delta W_{\mathrm{N}}= & f_{\mathrm{s}} b_{\mathrm{s}}(1-r)\left(P_{\mathrm{H}}+P_{\mathrm{D}}\right) \\
& -f_{\mathrm{s}} 0 \\
& +f_{\mathrm{h}} b_{\mathrm{h}}(1-r)\left(P_{\mathrm{H}}+P_{\mathrm{D}}\right) \\
& -f_{\mathrm{h}} 0
\end{aligned}
$$


A Nonhelper always asks for help, but will receive it only when asking a non-relative (since relatives employ the same strategy, and thus never help), which occurs with probability $1-r$, who employs one of the helping strategies, which occurs with probability $\left(P_{\mathrm{H}}+P_{\mathrm{D}}\right)$. A Nonhelper never provides care.

The fitness benefit for Honest Helpers is

$$
\begin{aligned}
\Delta W_{\mathrm{H}}= & f_{\mathrm{s}} b_{\mathrm{s}}\left(r+(1-r)\left(P_{\mathrm{H}}+P_{\mathrm{D}}\right)\right) \\
& -f_{\mathrm{s}} c \\
& +f_{\mathrm{h}} 0 \\
& -f_{\mathrm{h}} c(1-r)\left(P_{\mathrm{N}}+P_{\mathrm{D}}\right)
\end{aligned}
$$

An Honest Helper will be helped when sick also by a relative, increasing the probability of receiving care when sick to $r+(1-r)\left(P_{\mathrm{H}}+P_{\mathrm{D}}\right)$, while she never asks for help when healthy. An Honest Helper always provides care when asked, and will always be asked if the recipient is sick. If the recipient is healthy, only a non-relative who employs one of the always asking strategies will use the opportunity to ask for health, which occurs with probability $(1-r)\left(P_{\mathrm{N}}+P_{\mathrm{D}}\right)$.

Finally, for Deceptive Helpers, we have

$$
\begin{aligned}
\Delta W_{\mathrm{D}}= & f_{\mathrm{s}} b_{\mathrm{s}}\left(r+(1-r)\left(P_{\mathrm{H}}+P_{\mathrm{D}}\right)\right) \\
& -f_{\mathrm{s}} c \\
& +f_{\mathrm{h}} b_{\mathrm{h}}\left(r+(1-r)\left(P_{\mathrm{H}}+P_{\mathrm{D}}\right)\right) \\
& -f_{\mathrm{h}} c\left(r+(1-r)\left(P_{\mathrm{N}}+P_{\mathrm{D}}\right)\right)
\end{aligned}
$$

A Deceptive Helper is not different to an Honest Helper when sick. Given the opportunity, a Deceptive Helper will ask for help also when healthy, and has the same probability to receive it as when sick. Contrasting to Honest Helper, a Deceptive Helper will be asked 
for help by a healthy relative, increasing the probability of providing care for healthy to $r+(1-r)\left(P_{\mathrm{N}}+P_{\mathrm{D}}\right)$.

\section{Evolutionarily Stable Strategies}

Using these expressions for fitness we can investigate the conditions under which each strategy is resistant to invasion from rare mutants of the other strategies. Strategies which can resist invasion from rare mutant are referred to as Evolutionarily Stable Strategies (ESS), a concept introduced by Maynard Smith and Price (1973).

The invasion conditions are derived in the supplementary materials, and are summarized in Table 1.

Table 1: Conditions for when mutant can invade single resident strategy

\begin{tabular}{llll}
\hline Mutant $\backslash$ Resident & Nonhelper & Honest Helper & Deceptive Helper \\
\hline Nonhelper & & $\frac{b_{\mathrm{h}}}{c}>\frac{\frac{f_{\mathrm{s}}}{f_{\mathrm{h}}}}{1-r}\left(r \frac{b_{\mathrm{s}}}{c}-1\right)$ & $\frac{b_{\mathrm{h}}}{c}<\frac{1}{r}\left(1+\frac{f_{\mathrm{s}}}{f_{\mathrm{h}}}-\frac{f_{\mathrm{s}}}{f_{\mathrm{h}}} \frac{b_{\mathrm{s}}}{c}\right)$ \\
Honest Helper & $\frac{b_{\mathrm{s}}}{c}>\frac{f_{\mathrm{h}}}{f_{\mathrm{s}}}\left(\frac{1}{r}-1\right)+\frac{1}{r}$ & & $\frac{b_{\mathrm{h}}}{c}<r$ \\
Deceptive Helper & $\frac{b_{\mathrm{h}}}{c}>\frac{1}{r}\left(1+\frac{f_{\mathrm{s}}}{f_{\mathrm{h}}}-\frac{f_{\mathrm{s}}}{f_{\mathrm{h}}} \frac{b_{\mathrm{s}}}{c}\right)$ & $\frac{b_{\mathrm{h}}}{c}>r$ & \\
\hline
\end{tabular}

The outcomes of these conditions can be visualized as a map in parameter space. In the plots that follow, relatedness has been fixed at $r=0.25$ and the frequencies have been fixed at $f_{\mathrm{s}}=f_{\mathrm{h}}=0.25$, and we plot using the normalized parameters $\frac{b_{\mathrm{h}}}{c}$ and $\frac{b_{\mathrm{s}}}{c}$, since it is the ratio of cost-to-benefit that determines the evolutionary outcomes, not the absolute values. In the supplementary materials we show that qualitatively similar results hold for a range of $r, f_{\mathrm{s}}$ and $f_{\mathrm{h}}$ values.

In one of the limiting cases, when $c<r b_{\mathrm{h}}$ (which implies that $c<r b_{\mathrm{s}}$ since we assume 
$b_{\mathrm{h}}<b_{\mathrm{s}}$ ), we have from Hamilton's rule that Deceptive Helper is the only Evolutionarily Stable Strategy, in the other extreme case where $c>r b_{\mathrm{s}}$ (which again implies that $c>r b_{\mathrm{h}}$ ) Hamilton's rule gives Nonhelper as the only Evolutionarily Stable Strategy. The interesting cases are thus in the parameter range where $r b_{\mathrm{h}}<c<r b_{\mathrm{s}}$, that is, where helping the ill is evolutionarily viable, but helping the healthy is not.

As can be seen in Figure 1, there is a broad range of conditions where the possibility of deception undermines caregiving (i.e., where Nonhelpers can establish - the light regions, mainly the orange and yellow regions and to a lesser extent the dark orange region). Now we consider the potential impact of aversive treatments on these evolutionary outcomes. For simplicity we assume that an aversive treatment reduces the benefit of receiving care when healthy and when sick by equal measure. Under this assumption, and in the context of Figures 1 and 2, the introduction of an aversive treatment can be thought of as shifting a model's point in the parameter space downward and to the left at a 45 degree angle (i.e., to follow a straight line with slope 1, in the left direction, where the length of the shift is determined by the aversiveness of the treatment). Figure 2 highlights those regions in the original parameter space where caregiving is undermined by illness deception (i.e., the light regions, where Nonhelpers can establish), but where it is possible for caregiving to become an Evolutionarily Stable Strategy, via a judicious choice of the degree of aversiveness of the treatment. Figure S1 in the supplementary materials shows that to the extent that illness deception undermines caregiving, aversive medicines can help prevent this erosion. That is to say, aversive medicines play a more important role when illness deception is common.

\section{Empirical predictions}

Here we outline how the theory and model generate predictions both about when we would expect to see harmful medicines and how harmful we would expect them to be, and speculate 


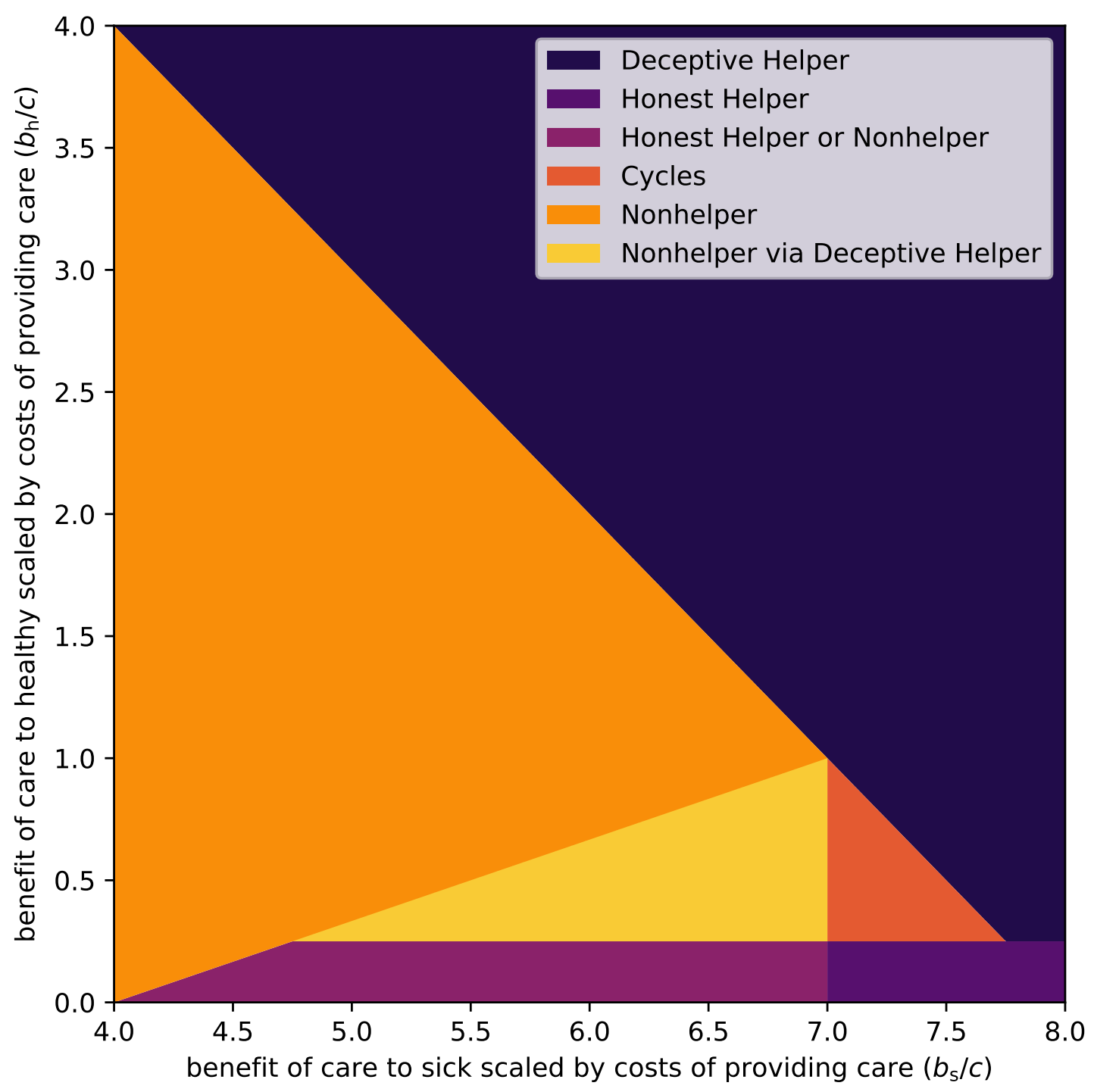

Figure 1: Evolutionarily Stable Strategies when relative benefit to sick $\left(b_{\mathrm{s}} / c\right)$ and relative benefit to healthy $\left(b_{\mathrm{h}} / c\right)$ vary. Relatedness is set to $r=0.25$, and opportunities for illness deception and legitimate care request occur with equal probability, $f_{\mathrm{s}}=f_{\mathrm{h}}=0.25$. The dark, blue/purple regions, are where helping strategies can be maintained: in the top right region Deceptive Helper dominates; in the bottom right region Honest Helper dominates; and in the bottom left region Honest Helper and Nonhelper are in a stalemate situation where both are evolutionarily stable, with neither being able to invade the other. Helping is not maintained in the light, yellow/orange regions: in the leftmost region Nonhelper dominates; in the central left triangular region, the dominance of Nonhelper is a direct result of the Deceptive Helper strategy being able to invade the Honest Helper strategy, paving the way for an invasion by Nonhelpers; and in the central right triangular region no strategy dominates, with Honest Helper being able to invade Nonhelper, which in turn is able to invade Deceptive Helper, which is in turn able to invade Honest Helper, and so on in a cycle. 


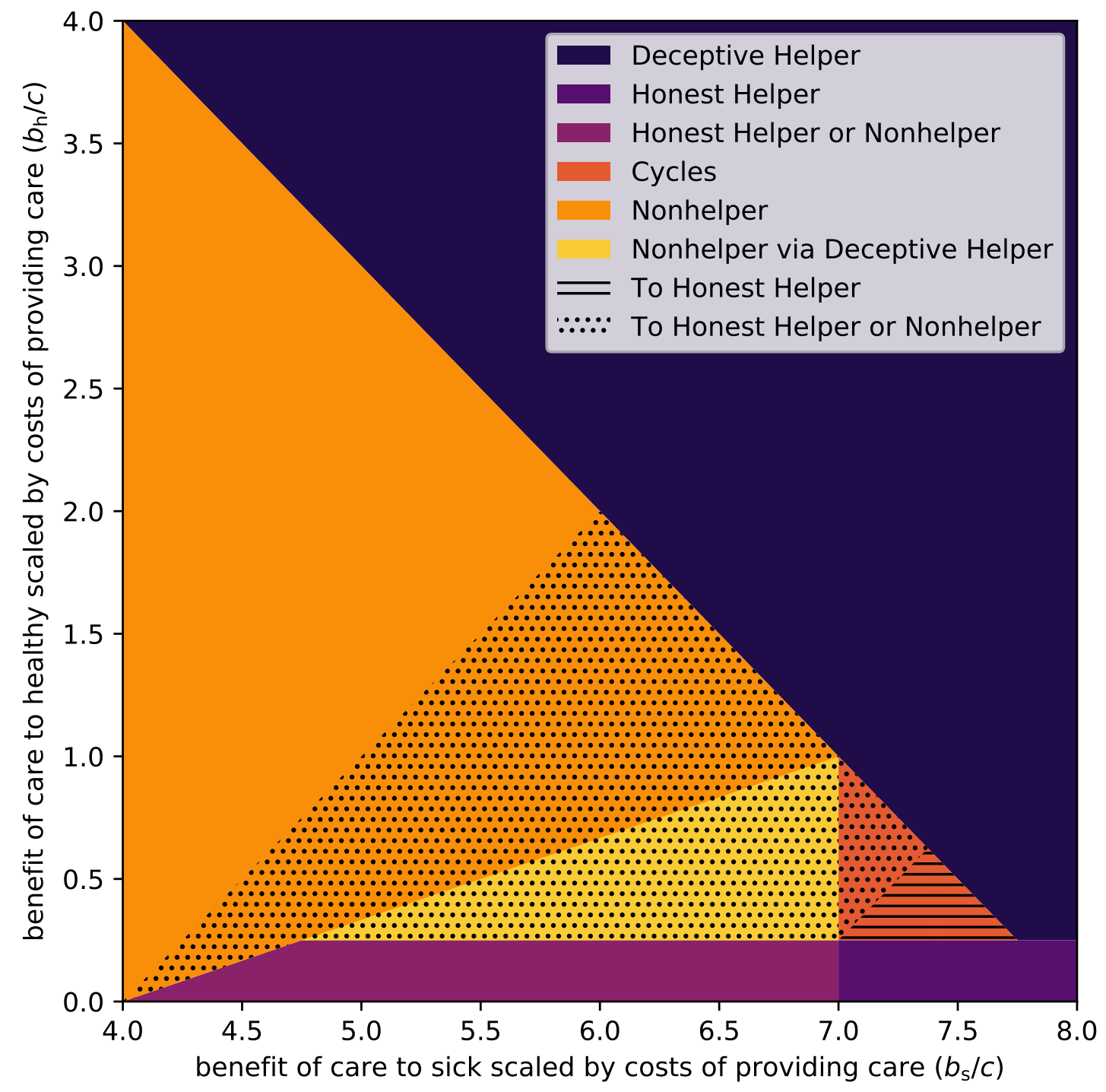

Figure 2: Evolutionarily Stable Strategies and potential helper ESSs when a harmful medical treatment is introduced. Relatedness is set to $r=0.25$, and opportunities for illness deception and legitimate care request occur with equal probability, $f_{\mathrm{s}}=f_{\mathrm{h}}=0.25$. The dotted region is where the dominance of Nonhelper (orange and yellow areas) can be eroded or cycling between Nonhelper and the other strategies (red area) can be stopped by aversive medicine, creating a stalemate situation where both Nonhelper and Honest Helper are evolutionarily stable. The lined area shows where aversive treatments can stop the cycling of strategies and make Honest Helper the sole Evolutionarily Stable Strategy. 
on how these predictions might be tested.

\section{How harmful should treatments be?}

In order to prevent illness deception from undermining caregiving, conditions must be set such that Nonhelper cannot invade. Table 1 shows that aversive medicine could potentially prevent Nonhelper from invading Honest Helper, but not from invading Deceptive Helper (since reducing $\frac{b_{\mathrm{h}}}{c}$ and $\frac{b_{\mathrm{s}}}{c}$ would decrease the left-hand side and increase the right-hand side of the inequality). However, an amount of harm could prevent Deceptive Helper from invading Honest Helper. Thus, there are two regions where aversive medicine could maintain caregiving: it can stop Nonhelpers from invading directly if $\frac{b_{\mathrm{h}}}{c}-a \leq \frac{f_{\mathrm{s}} / f_{\mathrm{h}}}{1-r}\left(r\left(\frac{b_{\mathrm{s}}}{c}-a\right)-1\right)$, and from invading by way of Deceptive Helper if $\frac{b_{\mathrm{h}}}{c}-a \leq r$, where $a \geq 0$ is the amount of harm of an aversive treatment. The smallest amount of $a$ that will meet the inequalities is thus

$$
a= \begin{cases}\frac{b_{\mathrm{h}}}{c}-r & \text { if Nonhelpers could invade only through Deceptive Helper } \\ \frac{\frac{b_{\mathrm{h}}}{c}-\frac{f_{\mathrm{s}} / f_{\mathrm{h}}}{1-r}\left(r \frac{b_{\mathrm{s}}}{c}-1\right)}{1-\frac{f_{\mathrm{s}} / f_{\mathrm{h}}}{1-r} r} & \text { if Nonhelpers could invade directly }\end{cases}
$$

Note that the denominator in the second expression is negative if (and only if) $\frac{r}{1-r}>\frac{f_{\mathrm{h}}}{f_{\mathrm{s}}}$, that is, when there are few opportunities to ask for help when healthy as compared to when sick, and/or most of these requests will be to relatives. For example, if $f_{\mathrm{s}} \leq f_{\mathrm{h}}$ and $r<0.5$, then the denominator is positive.

The first expression increases with $\frac{b_{\mathrm{h}}}{c}$, and so does the second when the denominator is positive, so we would expect to see more severe treatments when the benefits of illness deception are great. Therefore we predict that treatments will be more harmful in times of war among potential combatants relative to times of peace or to non-potential combatants. Similarly, we expect treatments to be more harmful in societies where people engage in dangerous foraging activities (e.g., hunting large mammals) than in societies where resource 
acquisition is safer.

On the other hand, to prevent Deceptive Helper from invading, we expect medicines to be less harmful when the denominator is large, that is when the costs to the caregiver are substantial. This is somewhat counter-intuitive - wouldn't a stronger deterrent be preferable when the costs of caregiving are large? - but it can be understood as a consequence of the fact that the cost of caregiving is disproportionately borne by relatives. Hence, from an inclusive fitness perspective, the costs to relatives of a request for care will not outweigh the benefits to self from that care. Finally, the closer the relatives, the more benign the treatment to stop Deceptive Helpers. Large cross-cultural ethnographic data bases may be useful in testing these within- and between-population predictions.

In the region where Nonhelpers can invade Honest Helpers directly, the aversiveness of medicine can either increase or decrease with costs and high relatedness, depending on the other variables. For example, if $\frac{f_{\mathrm{s}}}{f_{\mathrm{h}}} \frac{r}{1-r} b_{\mathrm{s}}>b_{\mathrm{h}}$, then medicines should be more harmful when the costs to the caregiver are large, while in the opposite case, they are expected to be less harmful. We refrain from going into further detail, the main point being that predictions are more complex when Nonhelpers can invade directly.

\section{When should harmful medicine be more common?}

Aversive medical treatments are expected to be more common when illness deception is possible. In situations where need is largely transparent - for example, when diagnostics are reliable or where the disease has obvious, familiar, symptoms that are difficult to fake then costly treatments are not needed. (Interestingly Steinkopf, 2015; Tiokhin, 2016 have argued that symptoms may have evolved in part for the purpose of signalling need.) To some extent, this is the situation that characterises contemporary medicine. Epidemic infectious diseases that infect large numbers of people and that have consistent symptomatology and consequences will also negate the need for harmful treatments. 
Generally speaking, cultures which deploy aversive treatments for ailments where the costs of providing and the benefits of receiving care, corresponding to those in the hatched parameter space of Figure 2, are less likely to have their caregiving practices undermined by illness deception. This has a number of implications. When $c$ is very low, caregivers have little to lose and much to gain by offering care freely. As $c$ increases, then we would expect costly treatments to become more common; in Figure 2 this is equivalent to a move from top right diagonally down and left into the hatched central area. In the real world a range of factors will influence $c$ including the caregiver's time or energy, food availability, or the scale of the care requested.

It follows that childhood illnesses are less likely to be treated with harmful medicines. Even when healthy, children's economic contribution is limited and hence the loss of their labour is a less significant problem. Moreover, children require substantial care independent of illness. Thus $c$ will generally be relatively low. For similar reasons, the elderly and infirm are also less likely to be treated with harmful medicines.

\section{Care without treatment?}

If a function of medical treatment is to legitimise one's request for care, then people should be less inclined to provide care to those who refuse treatment. For if potential illness deceivers could access care without treatment, then treatment's capacity to stabilise caregiving will evaporate. Thus, we make the final prediction that care will often be conditional on the acceptance of treatment. Experimental analysis and/or cross-sectional caregiving behaviour may be useful in assessing this hypothesis. 


\section{Discussion}

The judicious introduction of harmful treatments, in conjunction with effective caregiving, serves to broaden the range of conditions where caregiving is evolutionarily viable.

This model shows how unhealthy medical treatments can improve health by enabling access to very important peer care. There is a broad range of conditions, that is, relative cost-benefit ratios of receiving and providing care, where the possibility of illness deception renders caregiving evolutionarily inviable. We show, however, that the introduction of aversive medicine that reduces the benefit of receiving care, both for the truly ill and the illness deceivers, can in some cases transform the underlying strategic situation so that caregiving becomes evolutionarily viable where previously it was not. Intuitively this is possible because the benefit of care for the truly sick is assumed to be greater than the benefit of care for the illness deceiver. The model shows that there is scope for the benefit of care to be reduced for both the ill and the illness deceivers in such a way that illness deception is no longer the evolutionarily dominant strategy, allowing caregiving the chance to flourish. Thus the results presented here suggest that harmful treatments may spread, counter-intuitively, because they enable access to beneficial care, and we expect the average net effect of "harmful" treatments be positive in many circumstances.

Although the model above analyses treatments as if they were genetic traits, medicine is clearly a cultural phenomenon. However, there are several processes by which genetic fitness could translate into cultural fitness. Once a harmful medical practice emerges in a community, people who accept/demand the use of this signal will, on average, have better health than those who reject it. Better health translates into more, healthier children, and thus if medical beliefs are passed from parent to child, its frequency will increase within the group. Moreover, people are probably more inclined to learn from healthy peers and parent/elders than from the ill. Thus oblique and horizontal transmission may also facilitate trait spread. Nonetheless, our simplifying assumption that learning/cultural-evolutionary processes will lead to similar 
outcomes to the more abstracted genetic processes has been questioned (Fawcett, Hamblin, \& Giraldeau, 2013).

Results of our model demonstrate that costly medicine could plausibly function to legitimise illness and facilitate access to care. Qualitative research suggests that some patients see medical treatments in exactly this light. In particular, people with illnesses which leave little or no discernible mark on the body may embrace harmful medicine. In a study of chronic pain sufferers, Kleinman (1988, cited in Glenton (2003)) reports:

The surgeries have had one clearly positive effect, in Howie's view. They have created icons of his travail, scars that he can show people, that he can touch himself to assure himself that there is something 'physically wrong' with his back. After each of his surgeries, he felt that his family, fellow police officers, and doctors became more sympathetic. As he contemplates yet another major surgical procedure, this latent social function of surgery is a large part of the decision making, since his overall judgement about the surgeries is that they have made things worse.

However, the usefulness of harmful medicine is not dependent on people understanding its functional role. We suggest that over many generations, harmful medicine spreads within a community because people who use it end up healthier (and having healthier kin) than people who do not. In fact, "deterrent" medicine may work better when its true function is hidden. If the message component were obvious, skilled illness deceivers might circumvent the treatment through persuasion and appeals to other kinds of evidence that purport to demonstrate their illness. Those who suspect illness deception would need to make an explicit accusation, an act likely to damage relationships, whether or not illness deception is taking place. If belief-in-effectiveness is the proximate mechanism, then we might expect medicines to be employed in contexts where there is little signalling value (e.g., further treatment of seriously and obviously ill people). 
The current model has no bearing on the spread of beneficial/effective treatments. Treatment benefits and harms are orthogonal rather than being points along a single dimension: a single treatment can be both harmful and helpful (e.g., surgery) and many treatments are only helpful to people who are sick. The arguments outlined here suggest that selection pressures may favour treatments higher in the harm dimension, but it is plausible that other selection pressures may favour treatments higher in the benefit dimension, particularly because the benefit of effective treatments is often only realised if the recipient is truly ill. Some specific theoretical work as well as general cultural evolution models suggest that treatments may also evolve towards helpfulness (Henrich \& Henrich, 2010; Tanaka, Kendal, \& Laland, 2009). However, our reading of medical history and cross-cultural data suggests this may not have been a common occurance.

We have examined the evolution of caregiving with a kin selection framework. However, reciprocity (Trivers, 1971) and reputation-based processes (Nowak \& Sigmund, 2005) may also shape the evolution of caregiving. By providing care, individuals may stand to benefit from reciprocal care during their own illnesses or at some other time if/when the target recuperates. Analyses of transfers in small scale societies suggest that food transfers can insure against future ill health or injury by creating an expectation or obligation of reciprocal transfer in future times (Gurven, 2004; Gurven, Allen-Arave, Hill, \& Hurtado, 2000). Providing caregiving for the ill may similarly buffer against future misfortune, though reciprocation is less likely if the target fails to recuperate.

The medicine as message model refines Talcot Parsons' concept of the sick role (1951). When someone occupies a sick role, they are (a) released from their social obligations, (b) not held morally responsible for the additional burden that this places on others, but (c) must do everything possible to exit the sick role, including taking any medications or treatments recommended by medical professionals. Parsons sees sickness as something that disturbs the web of mutual dependencies because the sick cannot meet their normal obligations and 
they place additional burdens on kith and kin. When one occupies the sick role one is therefore obligated to make a good faith effort to recover and this includes accepting all expert treatment recommendations. The medicine as message theory suggests that this obligation to undergo the trials of treatment helps to maintain the stability of the institution in the face of would-be deceivers; if diagnostic tools are unreliable, a treatment-optional sick role would be overwhelmed.

Some of the costs and benefits of caregiving/illness play out within groups rather than in dyads, as in our model. Illness deception can be used to free oneself from dangerous or unpleasant collective tasks, for example, warfare, community construction projects, or shared food production. The existence of harmful medicine treatments can help to remove one important form of defection in these social dilemmas. Communities with bad medicine will be communities with good cooperation, and this cooperative-advantage may also enhance the cultural fitness of these traits.

This work adds to a growing literature demonstrating that evolutionary thinking can add insight not just to medicine and biology, but also to the practice of medicine itself (De Barra, 2017; De Barra, Eriksson, \& Strimling, 2014; Fabrega, 2011; Miton \& Mercier, 2015; Miton, Claidière, \& Mercier, 2015; Steinkopf, 2017; Tanaka et al., 2009). Much of this work has focussed on detrimental or harmful treatments, though see Tanaka et al. (2002). Miton et al. show that people's intuition about what makes a good treatment can shape the cultural evolutionary processes, because intuitive treatments tend to be recalled and retold more accurately and with fewer omissions. This gives them an advantage as they spread through networks of individuals. However, people's intuition about good treatments - for example, removal of harmful substances through purging or bloodletting - rarely correspond with the biological reality (Boudry, Blancke, \& Pigliucci, 2015), and so culturally successful treatments are rarely biomedical successes. Miton et al.'s findings are helpful in explaining why harmful treatment across different cultures share common features. Findings from de Barra et al. 
may help to explain why harmful medical treatments continue to maintain a good reputation (De Barra, 2017; De Barra et al., 2014). An analysis of on-line reviews of multiple medical treatments suggests that people who have positive outcomes are more inclined to tell other people about their treatment than people who have average or negative outcomes. Much like the file drawer problem in science, this positively distorts the treatment's reputation.

The evolutionary analysis of placebos and symptoms developed by Steinkopf and others compliments our arguments (Steinkopf, 2015, 2016, 2017; Tiokhin, 2016). As in the present work, they emphasise that healing is enabled by care, and that sick individuals thus need to reliably communicate their condition to social partners. In consequence, they argue, the reduction in symptomatology that follows treatment occurs because that signalling role of symptoms has now been fulfilled. Thus placebo effects stem not from the proverbial sugar pill, but from the broader social context of the clinical encounter, a perspective emphasised by Moerman (2002). Indeed, the present work may explain why more invasive treatments appear to elicit a stronger placebo effect.

In conclusion, the medicine as message model presented here suggests an explanation to several puzzling questions of medical cultural evolution. Medicines served not just to cure disease but also to deter illness deception. Many treatments that are directly harmful may be indirectly beneficial in that they help to expand the range of circumstances in which valuable caregiving can persist.

\section{References}

Boudry, M., Blancke, S., \& Pigliucci, M. (2015). What makes weird beliefs thrive? The epidemiology of pseudoscience. Philosophical Psychology, 28(8), 1177-1198.

Boyd, R., Richerson, P. J., \& Henrich, J. (2013). The cultural evolution of technology: Facts 
and theories. Cultural Evolution: Society, Technology, Language, and Religion, 119-142.

Brännström, Å., Johansson, J., \& Festenberg, N. von. (2013). The Hitchhiker's Guide to Adaptive Dynamics. Games, 4(3), 304-328.

Byrne, R. W., \& Stokes, E. (2003). Can monkeys malinger? In Malingering and illness deception (pp. 54-67). Oxford University Press.

Chagnon, N. A. (2013). Noble savages: My life among two dangerous tribes-the yanomamo and the anthropologists. Simon; Schuster.

De Barra, M. (2017). Reporting bias inflates the reputation of medical treatments: A comparison of outcomes in clinical trials and online product reviews. Social Science $\&$ Medicine, 177, 248-255.

De Barra, M., Eriksson, K., \& Strimling, P. (2014). How feedback biases give ineffective medical treatments a good reputation. Journal of Medical Internet Research, 16(8).

Edgerton, R. B. (1992). Sick societies. Simon; Schuster.

Fabrega, H. J. (2011). Sickness and healing and the evolutionary foundations of mind and minding. Mens Sana Monogr., 169-182.

Fawcett, T. W., Hamblin, S., \& Giraldeau, L.-A. (2013). Exposing the behavioral gambit: The evolution of learning and decision rules. Behavioral Ecology, 24(1), 2-11.

Finlay, B. L., \& Syal, S. (2014). The pain of altruism. Trends in Cognitive Sciences, 18(12), $615-617$.

Gardner, A., \& West, S. A. (2006). Demography, altruism, and the benefits of budding. Journal of Evolutionary Biology, 19(5), 1707-1716.

Glenton, C. (2003). Chronic back pain sufferers - striving for the sick role. Social Science $\&$ 
Medicine, 57(11), 2243-2252.

Gun, R. T. (1990). The incidence and distribution of RSI in south australia 1980-81 to 1986-87. The Medical Journal of Australia, 153(7), 376-380.

Gurven, M. (2004). To give and to give not: The behavioral ecology of human food transfers. Behavioral and Brain Sciences, 27(4), 543-559.

Gurven, M., Allen-Arave, W., Hill, K., \& Hurtado, M. (2000). "It's a wonderful life": Signaling generosity among the ache of paraguay. Evolution and Human Behavior, 21(4), $263-282$.

Gurven, M., Stieglitz, J., Hooper, P. L., Gomes, C., \& Kaplan, H. (2012). From the womb to the tomb: The role of transfers in shaping the evolved human life history. Experimental Gerontology, 47(10), 807-813.

Halligan, P. W., Bass, C. M., \& Oakley, D. . (2003). How can organizations prevent illness deception among employees? Oxford University Press New York.

Hamilton, W. D. (1963). The evolution of altruistic behavior. The American Naturalist, $97(896), 354-356$.

Hardy, A. (2006). First do no harm. EMBO Reports, 7(12), 1199-1199.

Henrich, J., \& Henrich, N. (2010). The evolution of cultural adaptations: Fijian food taboos protect against dangerous marine toxins. Proceedings of the Royal Society B: Biological Sciences, 27r(1701), 3715-3724.

Hill, K., \& Hurtado, A. M. (2009). Cooperative breeding in south american hunter-gatherers. Proceedings of the Royal Society of London B: Biological Sciences, rspb20091061.

Hublin, J.-J. (2009). The prehistory of compassion. Proceedings of the National Academy of Sciences, $106(16)$, 6429-6430.

Kaplan, H., Hill, K., Lancaster, J., \& Hurtado, A. M. (2000). A theory of human life history 
evolution: Diet, intelligence, and longevity. Evolutionary Anthropology Issues News and Reviews, 9(4), 156-185.

Kleinman, A. (1988). Suffering, healing \& the human condition. New York, USA: Basic Book Publication, 18-30.

Loeser, J. D., Henderlite, S. E., \& Conrad, D. A. (1995). Incentive effects of workers' compensation benefits: A literature synthesis. Medical Care Research and Review, 52(1), $34-59$.

Marks, N. F. (1996). Caregiving across the lifespan: National prevalence and predictors. Family Relations, 27-36.

Maynard Smith, J. (1991). Honest signalling: The philip sidney game. Animal Behaviour, 42(6), 1034-1035.

Maynard Smith, J., \& Price, G. R. (1973). The logic of animal conflict. Nature, 246, 15.

Miton, H., \& Mercier, H. (2015). Cognitive obstacles to pro-vaccination beliefs. Trends in Cognitive Sciences, 19(11), 633-636.

Miton, H., Claidière, N., \& Mercier, H. (2015). Universal cognitive mechanisms explain the cultural success of bloodletting. Evolution and Human Behavior, 36 (4), 303-312.

Mittenberg, W., Patton, C., Canyock, E. M., \& Condit, D. C. (2002). Base rates of malingering and symptom exeggeration. Journal of Clinical and Experimental Neuropsychology, 24(8), 1094-1102.

Moerman, D. E. (2002). Meaning, medicine, and the "placebo effect" (Vol. 28). Cambridge University Press Cambridge.

Nicholson, K., \& Martelli, M. F. (2007). The effect of compensation status. In Causality of psychological injury (pp. 411-426). Springer.

Norman, G. R., Tugwell, P., \& Feightner, J. W. (1982). A comparison of resident performance 
on real and simulated patients. Academic Medicine, 57(9), 708-15.

Nowak, M. A., \& Sigmund, K. (2005). Evolution of indirect reciprocity. Nature, 437(7063), $1291-1298$.

Parsons, T. (1951). Illness and the role of the physician: A sociological perspective. American Journal of Orthopsychiatry, 21(3), 452-460.

Perkins, M., Howard, V. J., Wadley, V. G., Crowe, M., Safford, M. M., Haley, W. E., ... Roth, D. L. (2012). Caregiving strain and all-cause mortality: Evidence from the regards study. The Journals of Gerontology Series B: Psychological Sciences and Social Sciences, $68(4), 504-512$.

Rohling, M. L., Binder, L. M., \& Langhinrichsen-Rohling, J. (1995). Money matters: A meta-analytic review of the association between financial compensation and the experience and treatment of chronic pain. Health Psychology, 14(6), 537.

Schulz, R., \& Beach, S. R. (1999). Caregiving as a risk factor for mortality: The caregiver health effects study. JAMA, 282(23), 2215-2219.

Steinkopf, L. (2015). The signaling theory of symptoms an evolutionary explanation of the placebo effect. Evolutionary Psychology, 13(3), 1474704915600559.

Steinkopf, L. (2016). An evolutionary perspective on pain communication. Evolutionary Psychology, 14 (2), 1474704916653964.

Steinkopf, L. (2017). The social situation of sickness: An evolutionary perspective on therapeutic encounters. Evolutionary Psychological Science, 1-17.

Sugg, R. (2008). Corpse medicine: Mummies, cannibals, and vampires. The Lancet, 371 (9630), 2078-2079.

Sugiyama, L. S. (2004). Illness, injury, and disability among shiwiar forager-horticulturalists: Implications of health-risk buffering for the evolution of human life history. American Journal 
of Physical Anthropology, 123(4), 371-389.

Sugiyama, L. S., \& Chacon, R. (2000). Effects of illness and injury on foraging among the yora and shiwiar: Pathology risk as adaptive problem. Human Behavior and Adaptation: An Anthropological Perspective, 371-395.

Tanaka, M. M., Kendal, J. R., \& Laland, K. N. (2009). From traditional medicine to witchcraft: Why medical treatments are not always efficacious. PLoS One, 4(4), e5192.

Tanaka, M. M., Kumm, J., \& Feldman, M. W. (2002). Coevolution of pathogens and cultural practices: A new look at behavioral heterogeneity in epidemics. Theoretical Population Biology, 62(2), 111-119.

Taylor, P. D., \& Frank, S. A. (1996). How to Make a Kin Selection Model. Journal of Theoretical Biology, 180(1), 27-37.

Tiokhin, L. (2016). Do symptoms of illness serve signaling functions?(Hint: Yes). The Quarterly Review of Biology, 91(2), 177-195.

Trivers, R. L. (1971). The evolution of reciprocal altruism. The Quarterly Review of Biology, $46(1), 35-57$.

Vitaliano, P. P., Zhang, J., \& Scanlan, J. M. (2003). Is caregiving hazardous to one's physical health? A meta-analysis. Psychological Bulletin, 129(6), 946.

West, S. A., Pen, I., \& Griffin, A. S. (2002). Cooperation and competition between relatives. Science, 296(5565), 72-75.

Withey, A. (2013). Physick and the family: Health, medicine and care in wales, 1600-1750. Oxford University Press.

Wootton, D. (2006). Bad medicine: Doctors doing harm since hippocrates. OUP Oxford. 